\title{
Three-Level Laser Coupled to Squeezed Vacuum Reservoir
}

\author{
Menisha Alemu* \\ Department of Physics, Wolkite University, P. O. Box 07, Wolkite, Ethiopia
}

Received January 27, 2020; Revised April 24, 2020; Accepted April 27, 2020

\begin{abstract}
Copyright $\odot 2020$ by authors, all rights reserved. Authors agree that this article remains permanently open access under the terms of the Creative Commons Attribution License 4.0 International License
\end{abstract}

\begin{abstract}
We analyze the quantum properties of the light generated by a three-level laser with an open cavity and coupled to a two-mode squeezed vacuum reservoir via a single-port mirror. The three-level laser consists of three-level atoms available in an open cavity and pumped from the bottom to the top level by means of electron bombardment. Applying the large-time approximation scheme, we have obtained the steady-state solutions of the equations of evolution for the expectation values of the atomic operators and the quantum Langevin equations for the cavity mode operators. Using the resulting steady-state solutions, we have calculated the mean photon number, the variance of the photon number, and the quadrature variance for the two-mode cavity light. We have seen that the light generated by the three-level laser is in a squeezed state and the squeezing occurs in the plus quadrature. It so turns out that the maximum quadrature squeezing of the two-mode cavity light is $45.68 \%$ for $M_{0}=0.59$ and $N_{0}=0.27$ below the vacuum-state level. In addition, we have shown that the effect of the squeezed parameter is to increase the mean and variance of the photon number.
\end{abstract}

Keywords Operator dynamics, Photon statistics, Quadrature squeezing, Spontaneous emission

\section{Introduction}

Squeezed states of light have played a crucial role in the development of quantum physics. Squeezing is one of the nonclassical features of light that has been extensively studied by several authors [1, 2, 3, 4, 5, 6, 7, 8, 9, 10, 11, 12]. In squeezed light the noise in one quadrature is below the vacuum-state level at the expense of enhanced fluctuations in the other quadrature, with the product of the uncertainties in the two quadratures satisfying the uncertainty relation [9, 10]. Squeezed light has potential applications in low- noise optical communication and weak signal detection [10]. Squeezed light can be generated by various quantum optical processes such as subharmonic generations [1, 2, 3, 4, 5], four-wave mixing [2, 3], resonance fluorescence [1, 8], second harmonic generation [1, 2, 3], and three-level laser under certain conditions [1, 10]. A three-level laser is a quantum optical system in which light is generated by three-level atoms inside a cavity usually coupled to a vacuum reservoir via a single- port mirror. When a three-level atom in a cascade configuration makes a transition from the top to the bottom level via the intermediate level, two photons are generated. If the two photons have the same frequency, then the three-level atom is called degenerate three-level atom otherwise it is called nondegenerate. The squeezing and statistical properties of the light produced by three-level lasers when the atoms are initially prepared in a coherent superposition of the top and bottom levels or when these levels are coupled by strong coherent light have been studied by several authors [13, 14, 15, 16, 17, 18, 19, 20]. These authors have found that these quantum optical systems can generate squeezed light under certain conditions.

Moreover, Fesseha [9, 10] has studied the squeezing and the statistical properties of the light produced by a three-level laser with the atoms placed in a closed cavity and pumped by electron bombardment. He has shown that the maximum quadrature squeezing of the light generated by the laser, operating below threshold, is found to be $50 \%$ below the vacuumstate level. Moreover, he has also found that the quadrature squeezing of the output light is equal to that of the cavity light. On the other hand, this study shows that the local quadrature squeezing is greater than the global quadrature squeezing. He has also found that a large part of the total mean photon number is confined in a relatively small frequency interval. In addition, 

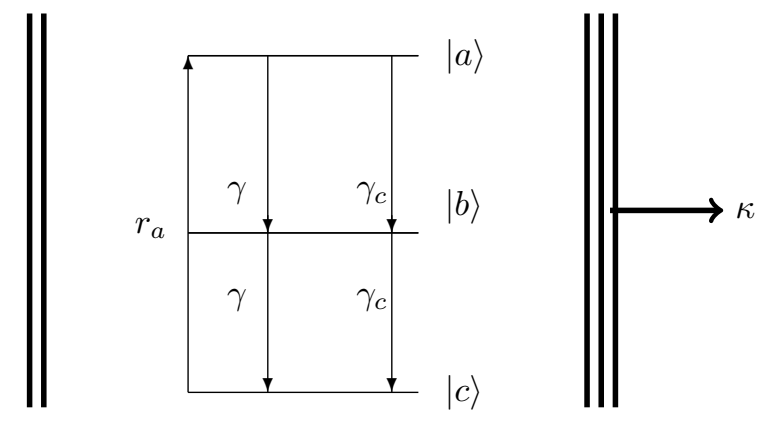

Figure 1. Schematic representation of a three-level laser coupled to a two-mode squeezed vacuum reservoir.

Fesseha [10] has studied the squeezing and the statistical properties of the light produced by a three-level laser with the atoms placed in a closed cavity and pumped by coherent light. He has shown that the maximum quadrature squeezing is $43 \%$ below the vacuum-state level, which is slightly less than the result found with electron bombardment.

In this paper, we seek to analyze the quantum properties of light emitted by three-level atoms available in an open cavity coupled to a squeezed vacuum reservoir via a single port-mirror and pumped from the bottom level to the top level by the means of electron bombardment. Thus taking into account the interaction of the three-level atoms with a resonant cavity light and the damping of the cavity light by a vacuum reservoir, we obtain the photon statistics and the quadrature squeezing of the cavity light. We carry out our calculation by considering the interactions of the cavity mode with the thermal reservoir via a single port-mirror and the three-level atoms with the cavity mode as well as a vacuum reservoir.

\section{Operator dynamics}

We consider here the case in which $\mathrm{N}$ three-level atoms in a cascade configuration and available in an open cavity. We denote the top, intermediate, and bottom levels of these atoms by $|a\rangle_{k},|b\rangle_{k}$, and $|c\rangle_{k}$, respectively. We prefer to call the light emitted from the top level light mode $a$ and the one emitted from the intermediate level light mode $b$. We carry out our analysis with light modes $a$ and $b$ having the same or different frequencies. In addition, we assume that light modes $a$ and $b$ to be at resonance with the two transitions $|a\rangle_{k} \rightarrow|b\rangle_{k}$ and $|b\rangle_{k} \rightarrow|c\rangle_{k}$, with direct transition between $|a\rangle_{k}$ and $|c\rangle_{k}$ to be electric-dipole forbidden. The interaction of a three-level atoms with cavity modes $a$ and $b$ can be described at resonance by the Hamiltonian [10]

$$
\hat{H}=i g\left(\hat{\sigma}_{a}^{\dagger k} \hat{a}-\hat{a}^{\dagger} \hat{\sigma}_{a}^{k}+\hat{\sigma}_{b}^{\dagger k} \hat{b}-\hat{b}^{\dagger} \hat{\sigma}_{b}^{k}\right),
$$

where

$$
\hat{\sigma}_{a}^{k}=|b\rangle_{k k}\langle a|,
$$

and

$$
\hat{\sigma}_{b}^{k}=|c\rangle_{k k}\langle b|
$$

are lowering atomic operators, $\hat{a}$ and $\hat{b}$ are the annihilation operators for the cavity modes, $\mathrm{g}$ is the coupling constant between the atom and the cavity modes. We assume that the cavity modes are coupled to a two-mode squeezed vacuum reservoir via a single-port mirror.

The quantum Langevin equations for the operators $\hat{a}$ and $\hat{b}$ are given by [9, 10]

$$
\begin{gathered}
\frac{d \hat{a}}{d t}=-\frac{\kappa}{2} \hat{a}-i[\hat{a}, \hat{H}]+\hat{F}_{a}(t), \\
\frac{d \hat{b}}{d t}=-\frac{\kappa}{2} \hat{b}-i[\hat{b}, \hat{H}]+\hat{F}_{b}(t),
\end{gathered}
$$

where $\kappa$ is the cavity damping constant and $\hat{F}_{a}(t)$ and $\hat{F}_{b}(t)$ are noise operators associated with squeezed vacuum reservoir and having the following correlation properties:

$$
\begin{gathered}
\left\langle\hat{F}_{a}(t)\right\rangle=\left\langle\hat{F}_{b}(t)\right\rangle=0, \\
\left\langle\hat{F}_{a}^{\dagger}(t) \hat{F}_{a}\left(t^{\prime}\right)\right\rangle=\left\langle\hat{F}_{b}^{\dagger}(t) \hat{F}_{b}\left(t^{\prime}\right)\right\rangle=\kappa N_{0} \delta\left(t-t^{\prime}\right), \\
\left\langle\hat{F}_{a}(t) \hat{F}_{a}^{\dagger}\left(t^{\prime}\right)\right\rangle=\left\langle\hat{F}_{b}(t) \hat{F}_{b}^{\dagger}\left(t^{\prime}\right)\right\rangle=\kappa\left(N_{0}+1\right) \delta\left(t-t^{\prime}\right),
\end{gathered}
$$




$$
\left\langle\hat{F}_{a}^{\dagger}(t) \hat{F}_{a}^{\dagger}\left(t^{\prime}\right)\right\rangle=\left\langle\hat{F}_{b}^{\dagger}(t) \hat{F}_{b}^{\dagger}\left(t^{\prime}\right)\right\rangle=\left\langle\hat{F}_{a}(t) \hat{F}_{a}\left(t^{\prime}\right)\right\rangle=\left\langle\hat{F}_{b}(t) \hat{F}_{b}\left(t^{\prime}\right)\right\rangle=-\kappa M_{0} \delta\left(t-t^{\prime}\right)
$$

With the aid of Eqs. (1), (4), and (5), one can easily establish that

$$
\begin{aligned}
& \frac{d \hat{a}}{d t}=-\frac{\kappa}{2} \hat{a}-g \hat{\sigma}_{a}^{k}+\hat{F}_{a}(t), \\
& \frac{d \hat{b}}{d t}=-\frac{\kappa}{2} \hat{b}-g \hat{\sigma}_{b}^{k}+\hat{F}_{b}(t) .
\end{aligned}
$$

Furthermore, the master equation for a three-level atom interacting with a squeezed vacuum reservoir is given by [10]

$$
\begin{aligned}
\frac{d \hat{\rho}}{d t} & =-i[\hat{H}, \hat{\rho}]+\frac{\gamma}{2}\left[2 \hat{\sigma}_{a}^{k} \hat{\rho} \hat{\sigma}_{a}^{\dagger k}-\hat{\sigma}_{a}^{\dagger k} \hat{\sigma}_{a}^{k} \hat{\rho}-\hat{\rho} \hat{\sigma}_{a}^{\dagger k} \hat{\sigma}_{a}^{k}+2 \hat{\sigma}_{b}^{k} \hat{\rho} \hat{\sigma}_{b}^{\dagger k}-\hat{\sigma}_{b}^{\dagger k} \hat{\sigma}_{b}^{k} \hat{\rho}-\hat{\rho} \hat{\sigma}_{b}^{\dagger k} \hat{\sigma}_{b}^{k}\right] \\
& +\frac{\kappa}{2}\left(N_{0}+1\right)\left[2 \hat{a} \hat{\rho} \hat{a}^{\dagger}-\hat{a}^{\dagger} \hat{a} \hat{\rho}-\hat{\rho} \hat{a}^{\dagger} \hat{a}\right]+\frac{\kappa}{2} N_{0}\left[2 \hat{a}^{\dagger} \hat{\rho} \hat{a}-\hat{a} \hat{a}^{\dagger} \hat{\rho}-\hat{\rho} \hat{a} \hat{a}^{\dagger}\right] \\
& +\frac{\kappa}{2} M_{0}\left[2 \hat{a} \hat{\rho} \hat{a}-\hat{a}^{2} \hat{\rho}-\hat{\rho} \hat{a}^{2}+2 \hat{a}^{\dagger} \hat{\rho} \hat{a}^{\dagger}-\hat{a}^{\dagger 2} \hat{\rho}-\hat{\rho} \hat{a}^{\dagger 2}\right]
\end{aligned}
$$

where

$$
\begin{gathered}
N_{0}=\sinh ^{2}(r) \\
M_{0}=\sinh (r) \cosh (r)
\end{gathered}
$$

with $r$ being squeezed parameter and $\gamma$ is the spontaneous emission decay constant. We can rewrite Eq. (12) as

$$
\begin{aligned}
\frac{d \hat{\rho}}{d t} & =-i[\hat{H}, \hat{\rho}]+\frac{\gamma}{2}\left[2 \hat{\sigma}_{a}^{k} \hat{\rho} \hat{\sigma}_{a}^{\dagger k}-\hat{\eta}_{a}^{k} \hat{\rho}-\hat{\rho} \hat{\eta}_{a}^{k}+2 \hat{\sigma}_{b}^{k} \hat{\rho} \hat{\sigma}_{b}^{\dagger k}-\hat{\eta}_{b}^{k} \hat{\rho}-\hat{\rho} \hat{\eta}_{b}^{k}\right] \\
& +\frac{\kappa}{2}\left(N_{0}+1\right)\left[2 \hat{a} \hat{\rho} \hat{a}^{\dagger}-\hat{a}^{\dagger} \hat{a} \hat{\rho}-\hat{\rho} \hat{a}^{\dagger} \hat{a}\right]+\frac{\kappa}{2} N_{0}\left[2 \hat{a}^{\dagger} \hat{\rho} \hat{a}-\hat{a} \hat{a}^{\dagger} \hat{\rho}-\hat{\rho} \hat{a} \hat{a}^{\dagger}\right] \\
& +\frac{\kappa}{2} M_{0}\left[2 \hat{a} \hat{\rho} \hat{a}-\hat{a}^{2} \hat{\rho}-\hat{\rho} \hat{a}^{2}+2 \hat{a}^{\dagger} \hat{\rho} \hat{a}^{\dagger}-\hat{a}^{\dagger 2} \hat{\rho}-\hat{\rho} \hat{a}^{\dagger 2}\right],
\end{aligned}
$$

where

$$
\begin{aligned}
& \hat{\eta}_{a}^{k}=|a\rangle_{k k}\langle a|, \\
& \hat{\eta}_{b}^{k}=|b\rangle_{k k}\langle b| .
\end{aligned}
$$

Using Eq. (1), we can put Eq. (15) in the form

$$
\begin{aligned}
\frac{d \hat{\rho}}{d t} & =g\left[\hat{\sigma}_{a}^{\dagger k} \hat{a} \hat{\rho}-\hat{\rho} \hat{\sigma}_{a}^{\dagger k} \hat{a}+\hat{\sigma}_{b}^{\dagger k} \hat{b} \hat{\rho}-\hat{\rho} \hat{\sigma}_{b}^{\dagger k} \hat{b}-\hat{a}^{\dagger} \hat{\sigma}_{a}^{k} \hat{\rho}-\hat{b}^{\dagger} \hat{\sigma}_{b}^{k} \hat{\rho}+\hat{\rho} \hat{a}^{\dagger} \hat{\sigma}_{a}^{k}+\hat{\rho} \hat{b}^{\dagger} \hat{\sigma}_{b}^{k}\right] \\
& +\frac{\gamma}{2}\left[2 \hat{\sigma}_{a}^{k} \hat{\rho} \hat{\sigma}_{a}^{\dagger k}-\hat{\eta}_{a}^{k} \hat{\rho}-\hat{\rho} \hat{\eta}_{a}^{k}+2 \hat{\sigma}_{b}^{k} \hat{\rho} \hat{\sigma}_{b}^{\dagger k}-\hat{\eta}_{b}^{k} \hat{\rho}-\hat{\rho} \hat{\eta}_{b}^{k}\right] \\
& +\frac{\kappa}{2}\left(N_{0}+1\right)\left[2 \hat{a} \hat{\rho} \hat{a}^{\dagger}-\hat{a} \hat{a}^{\dagger} \hat{\rho}-\hat{\rho} \hat{a}^{\dagger} \hat{a}\right]+\frac{\kappa}{2} N_{0}\left[2 \hat{a}^{\dagger} \hat{\rho} \hat{a}-\hat{a} \hat{a}^{\dagger} \hat{\rho}-\hat{\rho} \hat{a} \hat{a}^{\dagger}\right] \\
& +\frac{\kappa}{2} M_{0}\left[2 \hat{a} \hat{\rho} \hat{a}-\hat{a}^{2} \hat{\rho}-\hat{\rho} \hat{a}^{2}+2 \hat{a}^{\dagger} \hat{\rho} \hat{a}^{\dagger}-\hat{a}^{\dagger 2} \hat{\rho}-\hat{\rho} \hat{a}^{\dagger 2}\right]
\end{aligned}
$$

Now applying the relation

$$
\frac{d}{d t}\langle\hat{A}\rangle=\operatorname{Tr}\left(\frac{d \hat{\rho}}{d t} \hat{A}\right)
$$

along with Eq. (18), we can easily establish that

$$
\begin{gathered}
\frac{d}{d t}\left\langle\hat{\sigma}_{a}^{k}\right\rangle=-\gamma\left\langle\hat{\sigma}_{a}^{k}\right\rangle+g\left[\left\langle\hat{\eta}_{b}^{k} \hat{a}\right\rangle-\left\langle\hat{\eta}_{a}^{k} \hat{a}\right\rangle+\left\langle\hat{b}^{\dagger} \hat{\sigma}_{c}^{k}\right\rangle\right] \\
\frac{d}{d t}\left\langle\hat{\sigma}_{b}^{k}\right\rangle=-\frac{\gamma}{2}\left\langle\hat{\sigma}_{b}^{k}\right\rangle+g\left[\left\langle\hat{\eta}_{c}^{k} \hat{b}\right\rangle-\left\langle\hat{\eta}_{b}^{k} \hat{b}\right\rangle+\left\langle\hat{a}^{\dagger} \hat{\sigma}_{c}^{k}\right\rangle\right] \\
\frac{d}{d t}\left\langle\hat{\sigma}_{c}^{k}\right\rangle=-\frac{\gamma}{2}\left\langle\hat{\sigma}_{c}^{k}\right\rangle+g\left[\left\langle\hat{\sigma}_{b}^{k} \hat{a}\right\rangle-\left\langle\hat{\sigma}_{a}^{k} \hat{b}\right\rangle\right] \\
\frac{d}{d t}\left\langle\hat{\eta}_{a}^{k}\right\rangle=-\gamma\left\langle\hat{\eta}_{a}^{k}\right\rangle+g\left[\left\langle\hat{\sigma}_{a}^{\dagger k} \hat{a}\right\rangle+\left\langle\hat{a}^{\dagger} \hat{\sigma}_{a}^{k}\right\rangle\right],
\end{gathered}
$$




$$
\begin{gathered}
\frac{d}{d t}\left\langle\hat{\eta}_{b}^{k}\right\rangle=\gamma\left[\left\langle\hat{\eta}_{a}^{k}\right\rangle-\left\langle\hat{\eta}_{b}^{k}\right\rangle\right]+g\left[\left\langle\hat{b}^{\dagger} \hat{\sigma}_{b}^{k}\right\rangle+\left\langle\hat{\sigma}_{b}^{\dagger k} \hat{b}\right\rangle-\left\langle\hat{\sigma}_{a}^{\dagger k} \hat{a}\right\rangle-\left\langle\hat{a}^{\dagger} \hat{\sigma}_{a}^{k}\right\rangle\right] \\
\frac{d}{d t}\left\langle\hat{\eta}_{c}^{k}\right\rangle=\gamma\left\langle\hat{\eta}_{b}^{k}\right\rangle-g\left[\left\langle\hat{b}^{\dagger} \hat{\sigma}_{b}^{k}\right\rangle+\left\langle\hat{\sigma}_{b}^{\dagger k} \hat{b}\right\rangle\right]
\end{gathered}
$$

where

$$
\hat{\sigma}_{c}^{k}=|c\rangle_{k k}\langle a|
$$

and

$$
\hat{\eta}_{c}^{k}=|c\rangle_{k k}\langle c|
$$

We see that Eqs. (20)- 25 are nonlinear differential equations and hence it is not possible to find exact time-dependent solutions of these equations. We intend to over come this problem by applying the large-time approximation [13]. Then using this approximation scheme, we get from Eqs. (10) and (11) the approximately valid relations

$$
\begin{aligned}
& \hat{a}=-\frac{2 g}{\kappa} \hat{\sigma}_{a}^{k}+\frac{2}{\kappa} \hat{F}_{a}(t), \\
& \hat{b}=-\frac{2 g}{\kappa} \hat{\sigma}_{b}^{k}+\frac{2}{\kappa} \hat{F}_{b}(t) .
\end{aligned}
$$

Evidently, these would turn out to be exact relations at steady state. Now combining Eqs. (28) and (29) with Eqs. (20)-(25), we get

$$
\begin{gathered}
\frac{d}{d t}\left\langle\hat{\sigma}_{a}^{k}\right\rangle=-\left[\gamma+\gamma_{c}\right]\left\langle\hat{\sigma}_{a}^{k}\right\rangle+\frac{2 g}{\kappa}\left[\left\langle\hat{\eta}_{b}^{k} \hat{F}_{a}(t)\right\rangle-\left\langle\hat{\eta}_{a}^{k} \hat{F}_{a}(t)\right\rangle+\left\langle\hat{F}_{b}^{\dagger}(t) \hat{\sigma}_{c}^{k}\right\rangle\right] \\
\frac{d}{d t}\left\langle\hat{\sigma}_{b}^{k}\right\rangle=-\left[\frac{\gamma}{2}+\frac{\gamma_{c}}{2}\right]\left\langle\hat{\sigma}_{b}^{k}\right\rangle+\frac{2 g}{\kappa}\left[\left\langle\hat{\eta}_{c}^{k} \hat{F}_{b}(t)\right\rangle-\left\langle\hat{\eta}_{b}^{k} \hat{F}_{b}(t)\right\rangle-\left\langle\hat{F}_{a}^{\dagger}(t) \hat{\sigma}_{c}^{k}\right\rangle\right] \\
\frac{d}{d t}\left\langle\hat{\sigma}_{c}^{k}\right\rangle=-\left[\frac{\gamma}{2}+\frac{\gamma_{c}}{2}\right]\left\langle\hat{\sigma}_{c}^{k}\right\rangle+\frac{2 g}{\kappa}\left[\left\langle\hat{\sigma}_{b}^{k} \hat{F}_{a}(t)\right\rangle-\left\langle\hat{\sigma}_{a}^{k} \hat{F}_{b}(t)\right\rangle\right] \\
\frac{d}{d t}\left\langle\hat{\eta}_{a}^{k}\right\rangle=-\left[\gamma+\gamma_{c}\right]\left\langle\hat{\eta}_{a}^{k}\right\rangle+\frac{2 g}{\kappa}\left[\left\langle\hat{\sigma}_{a}^{\dagger k} \hat{F}_{a}(t)\right\rangle+\left\langle\hat{F}_{a}^{\dagger}(t) \hat{\sigma}_{a}^{k}\right\rangle\right] \\
\frac{d}{d t}\left\langle\hat{\eta}_{b}^{k}\right\rangle=-\left[\gamma+\gamma_{c}\right]\left\langle\hat{\eta}_{b}^{k}\right\rangle+\left[\gamma+\gamma_{c}\right]\left\langle\hat{\eta}_{a}^{k}\right\rangle \\
+\frac{2 g}{\kappa}\left[\left\langle\hat{F}_{b}^{\dagger}(t) \hat{\sigma}_{b}^{k}\right\rangle+\left\langle\hat{\sigma}_{b}^{\dagger k} \hat{F}_{b}(t)\right\rangle-\left\langle\hat{\sigma}_{a}^{\dagger k} \hat{F}_{a}(t)\right\rangle-\left\langle\hat{F}_{a}^{\dagger}(t) \hat{\sigma}_{a}^{k}\right\rangle\right] \\
\frac{d}{d t}\left\langle\hat{\eta}_{c}^{k}\right\rangle=\left[\gamma+\gamma_{c}\right]\left\langle\hat{\eta}_{b}^{k}\right\rangle-\frac{2 g}{\kappa}\left[\left\langle\hat{F}_{b}^{\dagger}(t) \hat{\sigma}_{b}^{k}\right\rangle+\left\langle\hat{\sigma}_{b}^{\dagger k} \hat{F}_{b}(t)\right\rangle\right]
\end{gathered}
$$

where

$$
\gamma_{c}=\frac{4 g^{2}}{\kappa}
$$

is the stimulated emission decay constant.

We next proceed to find the expectation value of the product involving a noise operator and an atomic operator that appears in Eqs. (30) - 35). To this end, after removing the angular brackets, Eq. (33) can be rewritten as

$$
\frac{d}{d t} \hat{\eta}_{a}^{k}=-\left[\gamma+\gamma_{c}\right] \hat{\eta}_{a}^{k}+\frac{2 g}{\kappa}\left[\hat{\sigma}_{a}^{\dagger k} \hat{F}_{a}(t)+\hat{F}_{a}^{\dagger}(t) \hat{\sigma}_{a}^{k}\right]+\hat{f}_{a}(t)
$$

where $\hat{f}_{a}(t)$ is the noise operator associated with $\hat{\eta}_{a}$. A formal solution of this equation can be written as

$$
\hat{\eta}_{a}^{k}(t)=\hat{\eta}_{a}^{k}(0) e^{-\left(\gamma+\gamma_{c}\right) t}+\int_{0}^{t} e^{-\left(\gamma+\gamma_{c}\right)\left(t-t^{\prime}\right)}\left[\frac{2 g}{\kappa}\left[\hat{\sigma}_{a}^{\dagger k}\left(t^{\prime}\right) \hat{F}_{a}\left(t^{\prime}\right)+\hat{F}_{a}^{\dagger}\left(t^{\prime}\right) \hat{\sigma}_{a}^{k}\left(t^{\prime}\right)\right]+\hat{f}_{a}\left(t^{\prime}\right)\right] d t^{\prime} .
$$

Multiplying Eq. 38) on the right by $\hat{F}_{a}(t)$ and taking the expectation value of the resulting equation, we have

$$
\begin{aligned}
& \left\langle\hat{\eta}_{a}^{k}(t) \hat{F}_{a}(t)\right\rangle=\left\langle\hat{\eta}_{a}^{k}(0) \hat{F}_{a}(t)\right\rangle e^{-\left(\gamma+\gamma_{c}\right) t}+\int_{0}^{t} e^{-\left(\gamma+\gamma_{c}\right)\left(t-t^{\prime}\right)} \\
& {\left[\frac{2 g}{\kappa}\left[\left\langle\hat{\sigma}_{a}^{\dagger k}\left(t^{\prime}\right) \hat{F}_{a}\left(t^{\prime}\right) \hat{F}_{a}(t)\right\rangle+\left\langle\hat{F}_{a}^{\dagger}\left(t^{\prime}\right) \hat{\sigma}_{a}^{k}\left(t^{\prime}\right) \hat{F}_{a}(t)\right\rangle\right]+\left\langle\hat{f}_{a}\left(t^{\prime}\right) \hat{F}_{a}(t)\right\rangle\right] d t^{\prime}}
\end{aligned}
$$


Ignoring the noncommutativity of the atomic and noise operators and neglecting the correlation between $\hat{F}_{a}(t)$ and $\hat{\sigma}_{a}^{k}\left(t^{\prime}\right)$, assumed to be considerably small [6], one can write the approximately valid relations

$$
\begin{aligned}
\left\langle\hat{\sigma}_{a}^{\dagger k}\left(t^{\prime}\right) \hat{F}_{a}\left(t^{\prime}\right) \hat{F}_{a}(t)\right\rangle & =\left\langle\hat{\sigma}_{a}^{\dagger k}\left(t^{\prime}\right)\right\rangle\left\langle\hat{F}_{a}\left(t^{\prime}\right) \hat{F}_{a}(t)\right\rangle=0, \\
\left\langle\hat{F}_{a}^{\dagger}\left(t^{\prime}\right) \hat{\sigma}_{a}^{k}\left(t^{\prime}\right) \hat{F}_{a}(t)\right\rangle & =\left\langle\hat{\sigma}_{a}^{k}\left(t^{\prime}\right)\right\rangle\left\langle\hat{F}_{a}^{\dagger}\left(t^{\prime}\right) \hat{F}_{a}(t)\right\rangle=0, \\
\left\langle\hat{f}_{a}\left(t^{\prime}\right) \hat{F}_{a}(t)\right\rangle & =\left\langle\hat{f}_{a}\left(t^{\prime}\right)\right\rangle\left\langle\hat{F}_{a}(t)\right\rangle=0 .
\end{aligned}
$$

Now on account of these approximately valid relations along with the fact that a noise operator $\hat{F}$ at a certain time should not affect the atomic variable at earlier time, Eq. 39. takes the form

$$
\left\langle\hat{\eta}_{a}^{k}(t) \hat{F}_{a}(t)\right\rangle=0 .
$$

Following a similar procedure, one can also check that

$$
\begin{aligned}
\left\langle\hat{\eta}_{b}^{k}(t) \hat{F}_{a}(t)\right\rangle & =0, \\
\left\langle\hat{\eta}_{c}^{k}(t) \hat{F}_{b}(t)\right\rangle & =0, \\
\left\langle\hat{\eta}_{b}^{k}(t) \hat{F}_{b}(t)\right\rangle & =0, \\
\left\langle\hat{F}_{a}^{\dagger}(t) \hat{\sigma}_{a}^{k}(t)\right\rangle & =0, \\
\left\langle\hat{F}_{b}^{\dagger}(t) \hat{\sigma}_{b}^{k}(t)\right\rangle & =0 .
\end{aligned}
$$

We also take

$$
\left\langle\hat{F}_{a}^{\dagger}(t) \hat{\sigma}_{c}^{k}(t)\right\rangle=\left\langle\hat{F}_{b}^{\dagger}(t) \hat{\sigma}_{c}^{k}(t)\right\rangle=0 .
$$

With the aid of Eqs. (43)- 49, we rewrite Eqs. 30, 31), 33, 34, and 35) as

$$
\begin{gathered}
\frac{d}{d t}\left\langle\hat{\sigma}_{a}^{k}\right\rangle=-\left[\gamma+\gamma_{c}\right]\left\langle\hat{\sigma}_{a}^{k}\right\rangle, \\
\frac{d}{d t}\left\langle\hat{\sigma}_{b}^{k}\right\rangle=-\left[\frac{\gamma}{2}+\frac{\gamma_{c}}{2}\right]\left\langle\hat{\sigma}_{b}^{k}\right\rangle . \\
\frac{d}{d t}\left\langle\hat{\eta}_{a}^{k}\right\rangle=-\left[\gamma+\gamma_{c}\right]\left\langle\hat{\eta}_{a}^{k}\right\rangle, \\
\frac{d}{d t}\left\langle\hat{\eta}_{b}^{k}\right\rangle=-\left[\gamma+\gamma_{c}\right]\left\langle\hat{\eta}_{b}^{k}\right\rangle+\left[\gamma+\gamma_{c}\right]\left\langle\hat{\eta}_{a}^{k}\right\rangle, \\
\frac{d}{d t}\left\langle\hat{\eta}_{c}^{k}\right\rangle=\left[\gamma+\gamma_{c}\right]\left\langle\hat{\eta}_{b}^{k}\right\rangle .
\end{gathered}
$$

We note that Eqs. 50 - 54 represent the equation of evolution for the atomic operators in the absence of the pumping process. The pumping process must surely affect the dynamics of $\left\langle\hat{\eta}_{a}^{k}\right\rangle$ and $\left\langle\hat{\eta}_{c}^{k}\right\rangle$. We seek here to pump the atoms by electron bombardment. If $r_{a}$ represents the rate at which a single atom is pumped from the bottom to the top level, then $\left\langle\hat{\eta}_{a}^{k}\right\rangle$ increases at the rate of $r_{a}\left\langle\hat{\eta}_{c}^{k}\right\rangle$ and $\left\langle\hat{\eta}_{c}^{k}\right\rangle$ decreases at the same rate. In view of this, we rewrite Eqs. (52) and (54) as

$$
\begin{gathered}
\frac{d}{d t}\left\langle\hat{\eta}_{a}^{k}\right\rangle=-\left[\gamma+\gamma_{c}\right]\left\langle\hat{\eta}_{a}^{k}\right\rangle+r_{a}\left\langle\hat{\eta}_{c}^{k}\right\rangle, \\
\frac{d}{d t}\left\langle\hat{\eta}_{c}^{k}\right\rangle=\left[\gamma+\gamma_{c}\right]\left\langle\hat{\eta}_{b}^{k}\right\rangle-r_{a}\left\langle\hat{\eta}_{c}\right\rangle .
\end{gathered}
$$

We next sum Eqs. (50), (51), (53), 55), and (56) over the $\mathrm{N}$ three-level atoms, so that

$$
\begin{gathered}
\frac{d}{d t}\left\langle\hat{m}_{a}\right\rangle=-\left[\gamma+\gamma_{c}\right]\left\langle\hat{m}_{a}\right\rangle, \\
\frac{d}{d t}\left\langle\hat{m}_{b}\right\rangle=-\left[\frac{\gamma}{2}+\frac{\gamma_{c}}{2}\right]\left\langle\hat{m}_{b}\right\rangle, \\
\frac{d}{d t}\left\langle\hat{N}_{a}\right\rangle=-\left[\gamma+\gamma_{c}\right]\left\langle\hat{N}_{a}\right\rangle+r_{a}\left\langle\hat{N}_{c}\right\rangle, \\
\frac{d}{d t}\left\langle\hat{N}_{b}\right\rangle=-\left[\gamma+\gamma_{c}\right]\left\langle\hat{N}_{b}\right\rangle+\left[\gamma+\gamma_{c}\right]\left\langle\hat{N}_{a}\right\rangle,
\end{gathered}
$$




$$
\frac{d}{d t}\left\langle\hat{N}_{c}\right\rangle=\left[\gamma+\gamma_{c}\right]\left\langle\hat{N}_{b}\right\rangle-r_{a}\left\langle\hat{N}_{c}\right\rangle
$$

in which

$$
\begin{aligned}
& \hat{m}_{a}=\sum_{k=1}^{N} \hat{\sigma}_{a}^{k}, \\
& \hat{m}_{b}=\sum_{k=1}^{N} \hat{\sigma}_{b}^{k}, \\
& \hat{N}_{a}=\sum_{k=1}^{N} \hat{\eta}_{a}^{k}, \\
& \hat{N}_{b}=\sum_{k=1}^{N} \hat{\eta}_{b}^{k}, \\
& \hat{N}_{c}=\sum_{k=1}^{N} \hat{\eta}_{c}^{k},
\end{aligned}
$$

with the operators $\hat{N}_{a}, \hat{N}_{b}$, and $\hat{N}_{c}$ representing the number of atoms in the top, intermediate, and bottom levels. In addition, employing the completeness relation

$$
\hat{\eta}_{a}^{k}+\hat{\eta}_{b}^{k}+\hat{\eta}_{c}^{k}=\hat{I}
$$

we easily arrive at

$$
\left\langle\hat{N}_{a}\right\rangle+\left\langle\hat{N}_{b}\right\rangle+\left\langle\hat{N}_{c}\right\rangle=N
$$

Furthermore, applying the definition given by Eq. (2) and setting for any $\mathrm{k}$

$$
\hat{\sigma}_{a}^{k}=|b\rangle\langle a|,
$$

we have

$$
\hat{m}_{a}=N|b\rangle\langle a| .
$$

Following the same procedure, one can also check that

$$
\begin{aligned}
& \hat{m}_{b}=N|c\rangle\langle b|, \\
& \hat{m}_{c}=N|c\rangle\langle a|, \\
& \hat{N}_{a}=N|a\rangle\langle a|, \\
& \hat{N}_{b}=N|b\rangle\langle b|, \\
& \hat{N}_{c}=N|c\rangle\langle c|,
\end{aligned}
$$

where

$$
\hat{m}_{c}=\sum_{k=1}^{N} \hat{\sigma}_{c}^{k}
$$

Moreover, using the definition

$$
\hat{m}=\hat{m}_{a}+\hat{m}_{b}
$$

and taking into account Eqs. (70)- (75), it can be readily established that

$$
\begin{gathered}
\hat{m}^{\dagger} \hat{m}=N\left(\hat{N}_{a}+\hat{N}_{b}\right), \\
\hat{m} \hat{m}^{\dagger}=N\left(\hat{N}_{b}+\hat{N}_{c}\right), \\
\hat{m}^{2}=N \hat{m}_{c} .
\end{gathered}
$$

With the aid of Eq. 68, one can put Eq. 59] in the form

$$
\frac{d}{d t}\left\langle\hat{N}_{a}\right\rangle=-\left[\gamma+\gamma_{c}+r_{a}\right]\left\langle\hat{N}_{a}\right\rangle+r_{a}\left[N-\left\langle\hat{N}_{b}\right\rangle\right]
$$


Applying the large-time approximation scheme to Eq. 60], we get

$$
\left\langle\hat{N}_{b}\right\rangle=\left\langle\hat{N}_{a}\right\rangle .
$$

Thus on taking into account this result, Eq. 81) can be written as

$$
\frac{d}{d t}\left\langle\hat{N}_{a}\right\rangle=-\left[\gamma+\gamma_{c}+2 r_{a}\right]\left\langle\hat{N}_{a}\right\rangle+N r_{a} .
$$

The steady-state solution of Eq. 83 is expressible as

$$
\left\langle\hat{N}_{a}\right\rangle=\frac{r_{a} N}{\gamma+\gamma_{c}+2 r_{a}} .
$$

Using the steady-state solution of Eq. (61) along with Eq. (82), we have

$$
\left\langle\hat{N}_{c}\right\rangle=\frac{\gamma+\gamma_{c}}{r_{a}}\left\langle\hat{N}_{a}\right\rangle
$$

On account of Eq. (84), Eq. (85) takes the form

$$
\left\langle\hat{N}_{c}\right\rangle=\frac{\left(\gamma+\gamma_{c}\right) N}{\gamma+\gamma_{c}+2 r_{a}} .
$$

For $r_{a}=0$, we see that $\left\langle\hat{N}_{a}\right\rangle=\left\langle\hat{N}_{b}\right\rangle=0$ and $\left\langle\hat{N}_{c}\right\rangle=N$. This result holds whether the atoms are initially in the top or bottom level.

In the presence of $\mathrm{N}$ three-level atoms, we rewrite Eq. [10] as [10]

$$
\frac{d \hat{a}}{d t}=-\frac{\kappa}{2} \hat{a}+\lambda \hat{m}_{a}+\beta \hat{F}_{a}(t),
$$

in which $\lambda$ and $\beta$ are constants whose values remain to be fixed. Applying Eq. [28, we get

$$
\left[\hat{a}, \hat{a}^{\dagger}\right]_{k}=\frac{4 g^{2}}{\kappa^{2}}\left(\hat{\eta}_{b}^{k}-\hat{\eta}_{a}^{k}\right)+\frac{4}{\kappa^{2}}\left[F_{a}, F_{a}^{\dagger}\right]
$$

and on summing over all atoms, we have

$$
\left[\hat{a}, \hat{a}^{\dagger}\right]=\frac{4 g^{2}}{\kappa^{2}}\left(\hat{N}_{b}-\hat{N}_{a}\right)+\frac{4 N}{\kappa^{2}}\left[F_{a}, F_{a}^{\dagger}\right]
$$

where

$$
\left[\hat{a}, \hat{a}^{\dagger}\right]=\sum_{k=1}^{N}\left[\hat{a}, \hat{a}^{\dagger}\right]_{k}
$$

stands for the commutator of $\hat{a}$ and $\hat{a}^{\dagger}$ when light mode $a$ is interecting with all the $\mathrm{N}$ three-level atoms. On the other hand, applying the large-time approximation to Eq. [87, one can easily find

$$
\left[\hat{a}, \hat{a}^{\dagger}\right]=N \frac{4 \lambda^{2}}{\kappa^{2}}\left(\hat{N}_{b}-\hat{N}_{a}\right)+\frac{4 \beta^{2}}{\kappa^{2}}\left[F_{a}, F_{a}^{\dagger}\right] .
$$

Thus on account of Eqs. (89) and (91), we see that

$$
\begin{aligned}
& \lambda= \pm \frac{g}{\sqrt{N}}, \\
& \beta= \pm \sqrt{N} .
\end{aligned}
$$

In view of Eqs. 92, and 93), Eq. 87) can be written as

$$
\frac{d \hat{a}}{d t}=-\frac{\kappa}{2} \hat{a}+\frac{g}{\sqrt{N}} \hat{m}_{a}+\sqrt{N} \hat{F}_{a}(t) .
$$

Following a similar procedure, one can also readily establish that

$$
\left[\hat{b}, \hat{b}^{\dagger}\right]=\frac{4 g^{2}}{\kappa^{2}}\left(\hat{N}_{c}-\hat{N}_{b}\right)+\frac{4 N}{\kappa^{2}}\left[F_{b}, F_{b}^{\dagger}\right],
$$




$$
\frac{d \hat{b}}{d t}=-\frac{\kappa}{2} \hat{b}+\frac{g}{\sqrt{N}} \hat{m}_{b}+\sqrt{N} \hat{F}_{b}(t) .
$$

Furthermore, in order to include the effect of pumping process, we rewrite Eqs. (57) and (58) as

$$
\begin{aligned}
\frac{d}{d t} \hat{m}_{a} & =-\frac{\mu}{2} \hat{m}_{a}+\hat{G}_{a}(t), \\
\frac{d}{d t} \hat{m}_{b} & =-\frac{\mu}{2} \hat{m}_{b}+\hat{G}_{b}(t)
\end{aligned}
$$

in which $\hat{G}_{a}(t)$ and $\hat{G}_{b}(t)$ are noise operators with vanishing mean and $\mu$ is a parameter whose value remains to be determined. Employing the relation

$$
\frac{d}{d t}\left\langle\hat{m}_{a}^{\dagger} \hat{m}_{a}\right\rangle=\left\langle\frac{d \hat{m}_{a}^{\dagger}}{d t} \hat{m}_{a}\right\rangle+\left\langle\hat{m}_{a}^{\dagger} \frac{d \hat{m}_{a}}{d t}\right\rangle
$$

along with Eq. 97, we easily find

$$
\frac{d}{d t}\left\langle\hat{m}_{a}^{\dagger} \hat{m}_{a}\right\rangle=-\mu\left\langle\hat{m}_{a}^{\dagger} \hat{m}_{a}\right\rangle+\left\langle\hat{m}_{a}^{\dagger} \hat{G}_{a}(t)\right\rangle+\left\langle\hat{G}_{a}^{\dagger}(t) \hat{m}_{a}\right\rangle,
$$

from which follows

$$
\frac{d}{d t}\left\langle\hat{N}_{a}\right\rangle=-\mu\left\langle\hat{N}_{a}\right\rangle+\frac{1}{N}\left[\left\langle\hat{m}_{a}^{\dagger} \hat{G}_{a}(t)\right\rangle+\left\langle\hat{G}_{a}^{\dagger}(t) \hat{m}_{a}\right\rangle\right] .
$$

Now comparison of Eqs. 83) and 101) shows that

$$
\mu=\gamma+\gamma_{c}+2 r_{a}
$$

and

$$
\left\langle\hat{m}_{a}^{\dagger} \hat{G}_{a}(t)\right\rangle+\left\langle\hat{G}_{a}^{\dagger}(t) \hat{m}_{a}\right\rangle=r_{a} N^{2} .
$$

We observe that Eq. 103 is equivalent to

$$
\left\langle\hat{G}_{a}^{\dagger}(t) \hat{G}_{a}\left(t^{\prime}\right)\right\rangle=r_{a} N^{2} \delta\left(t-t^{\prime}\right) .
$$

One can also easily verify that

$$
\left\langle\hat{G}_{a}(t) \hat{G}_{a}^{\dagger}\left(t^{\prime}\right)\right\rangle=\left(\gamma+\gamma_{c}\right) N^{2} \delta\left(t-t^{\prime}\right) .
$$

Furthermore, adding Eqs. (57) and (58), we have

$$
\frac{d}{d t}\langle\hat{m}\rangle=-\frac{1}{2}\left[\gamma+\gamma_{c}\right]\langle\hat{m}\rangle-\frac{1}{2}\left[\gamma+\gamma_{c}\right]\left\langle\hat{m}_{a}\right\rangle,
$$

where $\hat{m}$ is given by Eq. (77). Upon casting Eq. (106) into the form

$$
\frac{d}{d t} \hat{m}=-\frac{\mu}{2} \hat{m}-\frac{\mu}{2} \hat{m}_{a}+\hat{G}(t),
$$

one can also easily verify that $\mu$ has the value given by Eq. (102) and

$$
\left\langle\hat{G}^{\dagger}(t) \hat{G}\left(t^{\prime}\right)\right\rangle=r_{a} N^{2} \delta\left(t-t^{\prime}\right)
$$

On the other hand, assuming the atoms to be initial in the bottom level, the expectation value of the solution of Eq. (97) happens to be

$$
\left\langle\hat{m}_{a}(t)\right\rangle=0 .
$$

Hence the expectation value of the solution of Eq. (94) turns out to be

$$
\langle\hat{a}(t)\rangle=0
$$

In view of Eqs. (94) and (110), we claim that $\hat{a}(t)$ is a Gaussian variable with zero mean. One can also easily verify that

$$
\langle\hat{b}(t)\rangle=0
$$

Then on account of Eqs. (96) and (111), we realize that $\hat{b}(t)$ is a Gaussian variable with zero mean. 
Furthermore adding Eqs. (110) and (111), we obtain

$$
\langle\hat{c}\rangle=0
$$

where

$$
\hat{c}=\hat{a}+\hat{b}
$$

In addition, adding Eqs.(94) and (96), we get

$$
\frac{d \hat{c}}{d t}=-\frac{\kappa}{2} \hat{c}+\frac{g}{\sqrt{N}} \hat{m}+\sqrt{N} \hat{F}_{c}(t),
$$

where

$$
\hat{F}_{c}(t)=\hat{F}_{a}(t)+\hat{F}_{b}(t)
$$

and $\hat{m}$ is given by Eq. 77). One can also easily check that

$$
\begin{gathered}
\left\langle\hat{F}_{c}(t)\right\rangle=0, \\
\left\langle\hat{F}_{c}^{\dagger}(t) \hat{F}_{c}\left(t^{\prime}\right)\right\rangle=2 N_{0} \kappa \delta\left(t-t^{\prime}\right), \\
\left\langle\hat{F}_{c}^{\dagger}(t) \hat{F}_{c}^{\dagger}\left(t^{\prime}\right)\right\rangle=\left\langle\hat{F}_{c}(t) \hat{F}_{c}(t)\right\rangle=-2 \kappa M_{0} \delta\left(t-t^{\prime}\right), \\
\left\langle\hat{F}_{c}(t) \hat{F}_{c}^{\dagger}\left(t^{\prime}\right)\right\rangle=2 \kappa\left(N_{0}+1\right) \delta\left(t-t^{\prime}\right) .
\end{gathered}
$$

In view of Eqs. (112) and (114), we see that $\hat{c}$ is a Gaussian variable with zero mean.

\section{Photon statistics}

In this section we wish to calculate the mean and variance of the photon number for the two-mode cavity light at steady state. To this end, using the relation

$$
\frac{d}{d t}\left\langle\hat{c}^{\dagger}(t) \hat{c}(t)\right\rangle=\left\langle\frac{d \hat{c}^{\dagger}(t)}{d t} \hat{c}(t)\right\rangle+\left\langle\hat{c}^{\dagger}(t) \frac{d \hat{c}(t)}{d t}\right\rangle
$$

along with Eq. (114), we readily find

$$
\begin{aligned}
& \left.\frac{d}{d t}\left\langle\hat{c}^{\dagger} t\right) \hat{c}(t)\right\rangle=-\kappa\left\langle\hat{c}^{\dagger}(t) \hat{c}(t)\right\rangle+\frac{g}{\sqrt{N}}\left[\left\langle\hat{c}^{\dagger}(t) \hat{m}(t)\right\rangle+\left\langle\hat{m}^{\dagger}(t) \hat{c}(t)\right\rangle\right] \\
& +\sqrt{N}\left[\left\langle\hat{F}_{c}^{\dagger}(t) \hat{c}(t)\right\rangle+\left\langle\hat{c}^{\dagger}(t) \hat{F}_{c}(t)\right\rangle\right] .
\end{aligned}
$$

Next we seek to evaluate $\left\langle\hat{c}^{\dagger}(t) \hat{m}(t)\right\rangle$. Applying the large-time approximation, one gets from Eq. (114) the approximately valid relation

$$
\hat{c}(t)=\frac{2 g}{\kappa \sqrt{N}} \hat{m}+\frac{2 \sqrt{N}}{\kappa} \hat{F}_{c}(t) .
$$

Multiplying the adjoint of Eq. (122) on the right by $\hat{m}(t)$ and taking the expectation value of the resulting expression, we get

$$
\left\langle\hat{c}^{\dagger}(t) \hat{m}(t)\right\rangle=\frac{2 g \sqrt{N}}{\kappa}\left[\left\langle\hat{N}_{a}(t)\right\rangle+\left\langle\hat{N}_{b}(t)\right\rangle\right]+\frac{2 \sqrt{N}}{\kappa}\left\langle\hat{F}_{c}^{\dagger}(t) \hat{m}(t)\right\rangle .
$$

We now proceed to evaluate $\left\langle\hat{F}_{c}^{\dagger}(t) \hat{m}(t)\right\rangle$. To this end, a formal solution of Eq. (107) can be written as

$$
\hat{m}(t)=\hat{m}(0) e^{-\frac{\mu}{2} t}+\int_{0}^{t} e^{-\frac{\mu}{2}\left(t-t^{\prime}\right)}\left[-\frac{\mu}{2} \hat{m}_{a}\left(t^{\prime}\right)+\hat{G}\left(t^{\prime}\right)\right] d t^{\prime} .
$$

Multiplying Eq. (124) on the left by $\hat{F}_{c}^{\dagger}(t)$ and taking the expectation value of the resulting expression, we have

$$
\left\langle\hat{F}_{c}^{\dagger}(t) \hat{m}(t)\right\rangle=\left\langle\hat{F}_{c}^{\dagger}(t) \hat{m}(0)\right\rangle e^{-\frac{\mu}{2} t}+\int_{0}^{t} e^{-\frac{\mu}{2}\left(t-t^{\prime}\right)}\left[-\frac{\mu}{2}\left\langle\hat{F}_{c}^{\dagger}(t) \hat{m}_{a}\left(t^{\prime}\right)\right\rangle+\left\langle\hat{F}_{c}^{\dagger}(t) \hat{G}\left(t^{\prime}\right)\right\rangle\right] d t^{\prime} .
$$

Taking into account the fact that a noise operator $\hat{F}$ at a certain time should not affect the atomic variable at earlier time and assuming that the cavity mode and atomic mode operators are not correlated, we get

$$
\left\langle\hat{F}_{c}^{\dagger}(t) \hat{m}(t)\right\rangle=0 .
$$




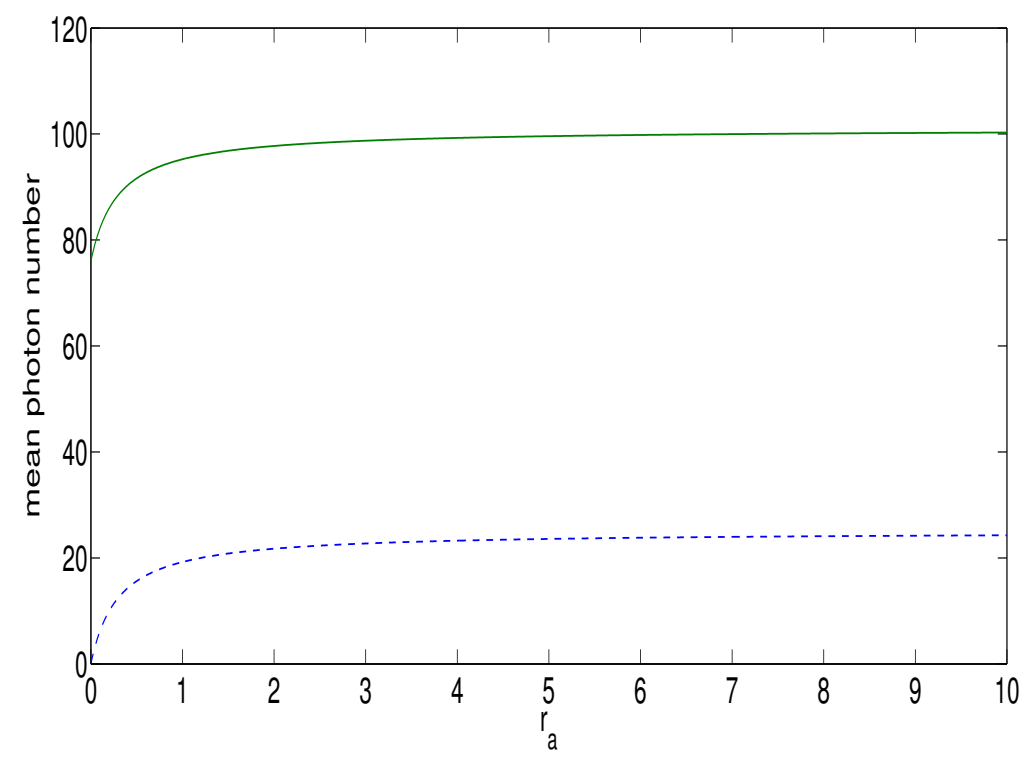

Figure 2. Plots of the mean photon number for the two-mode cavity light at steady state [Eq. [135] for $\kappa=0.8, \gamma_{c}=0.4, \gamma=0.2, N_{0}=0.2$ (solid curve), $N_{0}=0$ (dashed curve), and $N=50$.

On account of this result, Eq. (123) takes the form

$$
\left\langle\hat{c}^{\dagger}(t) \hat{m}(t)\right\rangle=\frac{2 g \sqrt{N}}{\kappa}\left[\left\langle\hat{N}_{a}(t)\right\rangle+\left\langle\hat{N}_{b}(t)\right\rangle\right]
$$

We next seek to evaluate $\left\langle\hat{F}_{c}^{\dagger}(t) \hat{c}(t)\right\rangle$. To this end, a formal solution of Eq. 114 can be written as

$$
\hat{c}(t)=\hat{c}(0) e^{-\frac{\kappa}{2} t}+\int_{0}^{t} e^{-\frac{\kappa}{2}\left(t-t^{\prime}\right)}\left[\frac{g}{\sqrt{N}} \hat{m}\left(t^{\prime}\right)+\sqrt{N} \hat{F}_{c}\left(t^{\prime}\right)\right] d t^{\prime} .
$$

Multiplying Eq. (128) on the left by $\hat{F}_{c}^{\dagger}(t)$ and taking the expectation value of the resulting expression, we get

$$
\left\langle\hat{F}_{c}^{\dagger}(t) \hat{c}(t)\right\rangle=\left\langle\hat{F}_{c}^{\dagger}(t) \hat{c}(0)\right\rangle e^{-\frac{\kappa}{2} t}+\int_{0}^{t} e^{-\frac{\kappa}{2}\left(t-t^{\prime}\right)}\left[\frac{g}{\sqrt{N}}\left\langle\hat{F}_{c}^{\dagger}(t) \hat{m}\left(t^{\prime}\right)\right\rangle+\sqrt{N}\left\langle\hat{F}_{c}^{\dagger}(t) \hat{F}_{c}\left(t^{\prime}\right)\right\rangle\right] d t^{\prime} .
$$

In view of Eqs. (117) and 126 along with the fact that a noise operator $\hat{F}$ at a certain time should not affect the atomic variable at earlier time, Eq. (129) becomes

$$
\left\langle\hat{F}_{c}^{\dagger}(t) \hat{c}(t)\right\rangle=\sqrt{N} N_{0} \kappa .
$$

Now on account of Eqs. (127) and (130) along with their complex conjugates, we can rewrite Eq. (121) as

$$
\frac{d}{d t}\left\langle\hat{c}^{\dagger}(t) \hat{c}(t)\right\rangle=-\kappa\left\langle\hat{c}^{\dagger}(t) \hat{c}(t)\right\rangle+\frac{4 g^{2}}{\kappa}\left[\left\langle\hat{N}_{a}(t)\right\rangle+\left\langle\hat{N}_{b}(t)\right\rangle\right]+2 \sqrt{N} N_{0} \kappa .
$$

The steady-state solution of this equation is expressible as

$$
\left\langle\hat{c}^{\dagger} \hat{c}\right\rangle=\frac{\gamma_{c}}{\kappa}\left[\left\langle\hat{N}_{a}\right\rangle+\left\langle\hat{N}_{b}\right\rangle\right]+2 N N_{0}
$$

Following a similar procedure, one can establish that

$$
\begin{gathered}
\left\langle\hat{c}^{\dagger}\right\rangle=\frac{\gamma_{c}}{\kappa}\left[\left\langle\hat{N}_{c}\right\rangle+\left\langle\hat{N}_{b}\right\rangle\right]+2 N\left(N_{0}+1\right), \\
\left\langle\hat{c}^{2}\right\rangle=\frac{\gamma_{c}}{\kappa}\left\langle\hat{m}_{c}\right\rangle-2 M_{0} N .
\end{gathered}
$$

In view of Eqs. (82), (84), and (86), Eqs. (132) and (133) can be rewritten as

$$
\left\langle\hat{c}^{\dagger} \hat{c}\right\rangle=\frac{\gamma_{c}}{\kappa}\left[\frac{2 N r_{a}}{\gamma+\gamma_{c}+2 r_{a}}\right]+2 N N_{0},
$$




$$
\left\langle\hat{c} \hat{c}^{\dagger}\right\rangle=\frac{\gamma_{c}}{\kappa}\left[\frac{\gamma+\gamma_{c}+r_{a}}{\gamma+\gamma_{c}+2 r_{a}}\right] N+2 N\left(N_{0}+1\right) .
$$

In the absence of squeezed parameter $(r=0)$, the mean photon number of the two-mode cavity light has the form

$$
\bar{n}_{c}=\frac{\gamma_{c}}{\kappa}\left(\frac{2 N r_{a}}{\gamma+\gamma_{c}+2 r_{a}}\right) .
$$

It can be seen from the plots in Fig. 2 that the mean photon number of two- mode cavity light is greater in the presence of squeezed parameter than in the absence of squeezed parameter. In other words, the effect of squeezed parameter is to increase the mean photon number.

Furthermore, the variance of the photon number for the two-mode cavity light is expressible as

$$
(\Delta n)^{2}=\left\langle\left(\hat{c}^{\dagger} \hat{c}\right)^{2}\right\rangle-\left\langle\hat{c}^{\dagger} \hat{c}\right\rangle^{2} .
$$

Using the fact that $\hat{c}$ is a Gaussian variable with zero mean, we readily get

$$
(\Delta n)^{2}=\left\langle\hat{c}^{\dagger} \hat{c}\right\rangle\left\langle\hat{c} \hat{c}^{\dagger}\right\rangle+\left\langle\hat{c}^{2 \dagger}\right\rangle\left\langle\hat{c}^{2}\right\rangle .
$$

We now proceed to calculate the expectation value of the atomic operator $\hat{m}_{c}$ following the approach presented in [10]. To this end, applying the identity given by Eq. 67), the state vector of a three-level atom can be put in the form

$$
\left|\psi_{k}\right\rangle=c_{a}\left|a_{k}\right\rangle+c_{b}\left|b_{k}\right\rangle+c_{c}\left|c_{k}\right\rangle
$$

in which

$$
\begin{aligned}
& c_{a}=\left\langle a_{k} \mid \psi_{k}\right\rangle, \\
& c_{b}=\left\langle b_{k} \mid \psi_{k}\right\rangle, \\
& c_{c}=\left\langle c_{k} \mid \psi_{k}\right\rangle .
\end{aligned}
$$

The state vector described by Eq. [140] can be used to determine the expectation value of an atomic operator formed by a pair of identical energy levels or by two distinct energy levels between which transition with the emission of a photon is dipole forbidden. One can thus readily establish that

$$
\begin{aligned}
& \left\langle\hat{\eta}_{a}^{k}\right\rangle=c_{a} c_{a}^{*}, \\
& \left\langle\hat{\eta}_{c}^{k}\right\rangle=c_{c} c_{c}^{*},
\end{aligned}
$$

and

$$
\left\langle\hat{\sigma}_{c}^{k}\right\rangle=c_{a} c_{c}^{*}
$$

We then see that

$$
\left|\left\langle\hat{\sigma}_{c}^{k}\right\rangle\right|^{2}=\left\langle\hat{\eta}_{a}^{k}\right\rangle\left\langle\hat{\eta}_{c}^{k}\right\rangle
$$

and on taking $\left|\left\langle\hat{\sigma}_{c}^{k}\right\rangle\right|$ to be real, we see that

$$
\left|\left\langle\hat{\sigma}_{c}^{k}\right\rangle\right|=\sqrt{\left\langle\hat{\eta}_{a}^{k}\right\rangle\left\langle\hat{\eta}_{c}^{k}\right\rangle}
$$

so that upon summing over $\mathrm{k}$ from 1 up to $\mathrm{N}$, we get

$$
\left\langle\hat{m}_{c}\right\rangle=\sqrt{\left\langle\hat{N}_{a}\right\rangle\left\langle\hat{N}_{c}\right\rangle} .
$$

On account of this, Eq. (134) takes the form

$$
\left\langle\hat{c}^{2}\right\rangle=\frac{\gamma_{c}}{\kappa} \sqrt{\left\langle\hat{N}_{a}\right\rangle\left\langle\hat{N}_{c}\right\rangle}-2 M_{0} N
$$

Now using Eq. (85), we have

$$
\left\langle\hat{c}^{2}\right\rangle=\frac{\gamma_{c}}{\kappa} \sqrt{\frac{\gamma+\gamma_{c}}{r_{a}}}\left\langle\hat{N}_{a}\right\rangle-2 M_{0} N .
$$

In view of Eqs. (132), (133), and (151), Eq. (139) becomes

$$
\begin{aligned}
& (\Delta n)^{2}=\left(\frac{\gamma_{c}}{\kappa}\left(\left\langle\hat{N}_{a}\right\rangle+\left\langle\hat{N}_{b}\right\rangle\right)+2 N N_{0}\right)\left(\frac{\gamma_{c}}{\kappa}\left(\left\langle\hat{N}_{b}\right\rangle+\left\langle\hat{N}_{c}\right\rangle\right)+2 N\left(N_{0}+1\right)\right) \\
& +\left(\frac{\gamma_{c}}{\kappa} \sqrt{\frac{\gamma+\gamma_{c}}{r_{a}}}\left\langle\hat{N}_{a}\right\rangle-2 N M_{0}\right)^{2}
\end{aligned}
$$


Finally, on account of Eqs. (82), (84), (85), and (86) along with Eq. (152), we arrive at

$$
\begin{aligned}
& (\Delta n)^{2}=\frac{1}{4} \bar{n}^{2}\left(\frac{3\left(\gamma+\gamma_{c}\right)}{r_{a}}+2\right)+2 \bar{n} N\left(1-N_{0}-\frac{\gamma+\gamma_{c}}{r_{a}} N_{0}-\sqrt{\frac{\gamma+\gamma_{c}}{r_{a}}} M_{0}\right) \\
& +N^{2}\left(\frac{\gamma+\gamma_{c}}{r_{a}} N_{0}^{2}-2 \sqrt{\frac{\gamma+\gamma_{c}}{r_{a}}} N_{0} M_{0}+4 M_{0}^{2}\right) .
\end{aligned}
$$

In the absence of squeezed parameter $(r=0)$, we readily find

$$
(\Delta n)^{2}=\frac{1}{4} \bar{n}^{2}(3 \eta+2)+2 N \bar{n},
$$

where

$$
\eta=\frac{\gamma+\gamma_{c}}{r_{a}} .
$$

Now inspection of Eq. (153) indicates that $(\Delta n)^{2}>\bar{n}$ and hence the photon statistics of the two-mode cavity light is superPoissonian. Our result shows that the photon number variance of the two-mode cavity light is greater than the one obtained by Fesseha [10]. This must be due to the reservoir noise operators.

\section{Quadrature squeezing}

We now proceed to calculate the quadrature squeezing of the two-mode cavity light in the entire frequency interval. To this end, the squeezing properties of the two-mode cavity light are described by two quadrature operators defined by

$$
\hat{c}_{+}=\hat{c}^{\dagger}+\hat{c}
$$

and

$$
\hat{c}_{-}=i\left(\hat{c}^{\dagger}-\hat{c}\right) .
$$

It can be readily established that [15]

$$
\left[\hat{c}_{-}, \hat{c}_{+}\right]=2 i \frac{\gamma_{c}}{\kappa}\left(\left\langle\hat{N}_{a}\right\rangle-\left\langle\hat{N}_{c}\right\rangle\right)-4 N i
$$

It then follows that [16]

$$
\Delta c_{+} \Delta c_{-} \geq \frac{\gamma_{c}}{\kappa}\left(\left\langle\hat{N}_{c}\right\rangle-\left\langle\hat{N}_{a}\right\rangle\right)+2 N .
$$

Upon setting $r_{a}=0$, we see that

$$
\Delta c_{+} \Delta c_{-} \geq \frac{\gamma_{c}}{\kappa} N+2 N .
$$

This represents the quadrature variance for two-mode vacuum state. The variance of the quadrature operator is expressible as

$$
\left(\Delta c_{ \pm}\right)^{2}= \pm\left\langle\left(\hat{c}^{\dagger} \pm \hat{c}\right)^{2}\right\rangle \mp\left[\left\langle\hat{c}^{\dagger}+\hat{c}\right\rangle\right]^{2}
$$

so that on account of Eq. 112, we have

$$
\left(\Delta c_{ \pm}\right)^{2}=\left\langle\hat{c}^{\dagger} \hat{c}\right\rangle+\left\langle\hat{c} \hat{c}^{\dagger}\right\rangle \pm\left\langle\hat{c}^{\dagger 2}\right\rangle \pm\left\langle\hat{c}^{2}\right\rangle
$$

Now employing Eqs. 68, 132, 133, and 1151, we arrive at

$$
\begin{aligned}
& \left(\Delta c_{+}\right)^{2}=\frac{\gamma_{c}}{\kappa}\left(N+\left\langle\hat{N}_{a}\right\rangle+2 \sqrt{\frac{\gamma+\gamma_{c}}{r_{a}}}\left\langle\hat{N}_{a}\right\rangle\right)+4 N N_{0}+2 N-4 M_{0} N, \\
& \left(\Delta c_{-}\right)^{2}=\frac{\gamma_{c}}{\kappa}\left(N+\left\langle\hat{N}_{a}\right\rangle-2 \sqrt{\frac{\gamma+\gamma_{c}}{r_{a}}}\left\langle\hat{N}_{a}\right\rangle\right)+4 N N_{0}+2 N+4 M_{0} N .
\end{aligned}
$$

Moreover, on setting $r_{a}$ and $N_{0}=M_{0}=0$ in Eqs. (163) and 164, we get

$$
\left(\Delta c_{+}\right)_{v}^{2}=\left(\Delta c_{-}\right)_{v}^{2}=\frac{\gamma_{c}}{\kappa} N+2 N
$$

This indeed represents the quadrature variance of a two-mode vacuum state. We seek to calculate the quadrature squeezing of the two-mode cavity light relative to the quadrature variance of the two-mode cavity vacuum state. We then define the quadrature squeezing of the two-mode cavity light by

$$
S=\frac{\left(\Delta c_{-}\right)_{v}^{2}-\left(\Delta c_{-}\right)^{2}}{\left(\Delta c_{-}\right)_{v}^{2}}
$$




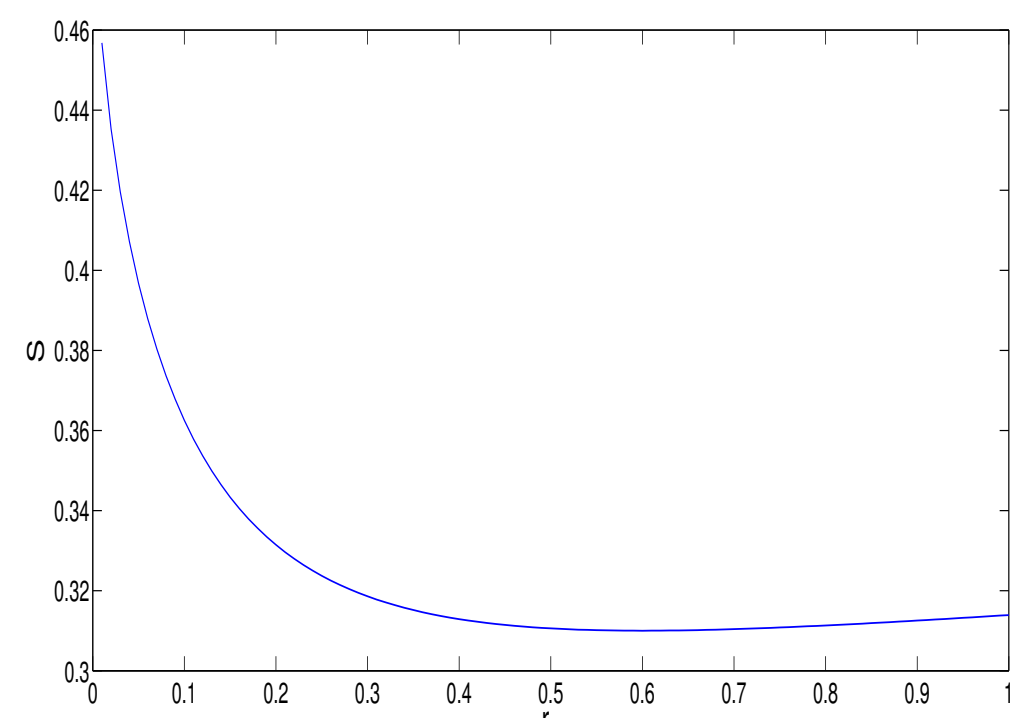

Figure 3. Plot of the quadrature squeezing at steady state, [Eq. [168] verstes $r_{a}$ for $\kappa=0.8, \gamma_{c}=0.4, \gamma=0.2, M_{0}=0.59$, and for $N_{0}=0.27$.

Now employing Eqs. (164) and (165), one can put Eq. 166) in the form

$$
S=\frac{4 \kappa\left(M_{0}-N_{0}\right)}{\left(\gamma_{c}+2 \kappa\right)}-\frac{\frac{\gamma_{c}}{\kappa}\left(2 \sqrt{\frac{\gamma+\gamma_{c}}{r_{a}}}\left\langle\hat{N}_{a}\right\rangle+\left\langle\hat{N}_{a}\right\rangle\right)}{\frac{\gamma_{c}}{\kappa} N+2 N} .
$$

On account of Eq. [84, Eq. [167) takes the form

$$
S=\frac{4 \kappa\left(M_{0}-N_{0}\right)}{\gamma_{c}+2 \kappa}-\frac{\gamma_{c}}{\gamma_{c}+2 \kappa}\left(\frac{2 \sqrt{\eta}+1}{\eta+2}\right) .
$$

We note that, unlike the mean photon number, the quadrature squeezing does not depend on the number of atoms. This implies that the quadrature squeezing of the cavity light is independent of the number of photons. The plot in Fig. 3 indicate that the maximum quadrature squeezing is $45.68 \%$ below the vacuum-state level and this occurs when the three-level laser is operating below threshold .

\section{Conclusion}

In this paper we have studied the statistical and squeezing properties of the light generated by three-level atoms available in an open cavity and pumped to the top level by electron bombardment at constant rate. Applying the large-time approximation scheme, we have obtained the steady-state solutions of the equations of evolution for the expectation values of the atomic operators and the quantum Langevin equations for the cavity mode operators.

Using the resulting steady-state solutions, we have calculated the mean photon number, the variance of the photon number, and the quadrature variance for the two-mode cavity light. We have seen that the light generated by the three-level laser is in a squeezed state and the squeezing occurs in the plus quadrature. It so turns out that the maximum quadrature squeezing of the two-mode cavity light is $45.68 \%$ below the vacuum-state level. Our result shows that the maximum quadrature squeezing is less than the one obtained by Fesseha [10]. This is due to the vacuum reservoir noise. In addition, we have shown that the effect of the squeezed parameter is to increase the mean and variance of the photon number.

\section{REFERENCES}

[1] M. S. Abdalla, E. M. Khalil, A. S.-F. Obada, J. Peřina, and J. Křepelka, "Quantum Statistical Characteristics of the Interaction between Two Two-Level Atoms and Radiation Field”, Eur. Phys. J. Plus. 130, 227 (2015).

[2] Fesseha Kassahun, Fundamentals of Quantum Optics (Lulu Press Inc., North Carolina, 2010).

[3] H. Nha, J. H. Lee, Y. T. Chough, S. W. Kim, and K. An, "Squeezing Enhancement by Damping in a Driven Atom-Cavity System”, J. Phys. Soc. Jpn. 71, 1615 (2002). 
[4] N. A. Ansari, Phys. Rev. A 48, 4686 (1993).

[5] Tewodros Y. Darge and Fesseha Kassahun, PMC Physics B, 1 (2010).

[6] Eyob Alebachew and K. Fesseha, Opt. Commun. 265, 314 (2006).

[7] Menisha Alemu, Spontaneous emission by three-level atoms pumped by electron bombardment,Universal Journal of Physics and Application 14(1): 11-22, 2020.

[8] N.A. Ansari, J. Gea-Banacloche, and M.S. Zubairy, Phys. Rev. A 41, 5179 (1990).

[9] Fesseha Kassahun, ArXiv:1105.1438v3 [quant-ph] 25 Sep 2012.

[10] Fesseha Kassahun, Refined Quantum Analysis of Light, Revised Edition (CreateSpace Independent Publishing Platform, 2016).

[11] C.A. Blockely and D.F. Walls, Phys. Rev. A 43, 5049 (1991).

[12] K. Fesseha, Phys. Rev. A 63, 33811 (2001).

[13] Fesseha, K. Stimulated Emission by Two-Level Atoms Pumped to the Upper Level. Optics Communications, 284, 1357-1363 (2011).

[14] N. Lu and S. Y. Zhu, Phys. Rev. A 40, 5735 (1989).

[15] Tewodros Yirgashewa, PhD Dissertation (Addis Ababa University, 2010).

[16] Menisha Alemu, PhD Dissertation (Addis Ababa University, 2018).

[17] D. F. Walls and J. G. Milburn, Quantum Optics (Springer-Verlag, Berlin, 1994).

[18] N. Lu and S. Y. Zhu, Phys. Rev. A 41, 2865 (1990).

[19] M. Xiao, L. A. Wu, H. J. Kimble, Phys. Rev. Lett. 59, 278 (1992).

[20] Sintayehu Tesfa, arXiv:0708.2815v1 [quant-ph] (2007). 